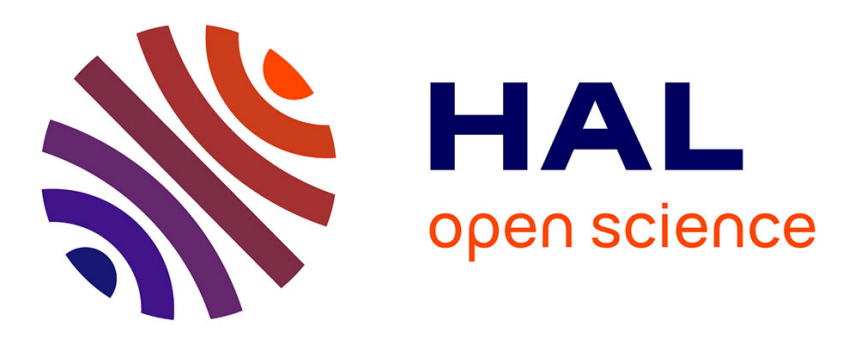

\title{
Theoretical approaches to laser spectroscopy in the presence of gravitational fields
}

Ch. J. Borde, J. Sharma, Ph. Tourrenc, Th. Damour

\section{To cite this version:}

Ch. J. Borde, J. Sharma, Ph. Tourrenc, Th. Damour. Theoretical approaches to laser spectroscopy in the presence of gravitational fields. Journal de Physique Lettres, 1983, 44 (24), pp.983-990. 10.1051/jphyslet:019830044024098300 . jpa-00232298

\section{HAL Id: jpa-00232298 https://hal.science/jpa-00232298}

Submitted on 1 Jan 1983

HAL is a multi-disciplinary open access archive for the deposit and dissemination of scientific research documents, whether they are published or not. The documents may come from teaching and research institutions in France or abroad, or from public or private research centers.
L'archive ouverte pluridisciplinaire HAL, est destinée au dépôt et à la diffusion de documents scientifiques de niveau recherche, publiés ou non, émanant des établissements d'enseignement et de recherche français ou étrangers, des laboratoires publics ou privés. 
Classification

Physics Abstracts

$32.70 \mathrm{~J}-04.80$

\title{
Theoretical approaches to laser spectroscopy in the presence of gravitational fields
}

\author{
Ch. J. Bordé, J. Sharma \\ Université Paris XIII, Laboratoire de Physique des Lasers (LA No 282), \\ av. J. B. Clément, 93430 Villetaneuse, France \\ $\mathrm{Ph}$. Tourrenc \\ Université Paris VI, Institut Henri Poincaré, \\ Laboratoire de Cosmologie et Gravitation Relativistes (E.R.A. No 533), \\ 11, rue P. et M. Curie, 75231 Paris Cedex 05, France
}

and Th. Damour

Observatoire de Meudon, Groupe d'Astrophysique Relativiste (ER No 176), 92190 Meudon, France

(Reçu le 3 août 1983, accepté le 27 octobre 1983)

\begin{abstract}
Résumé. - Nous présentons un cadre théorique général pour l'étude des processus d'optique nonlinéaire relatifs à un ensemble d'atomes accélérés. Nous montrons l'équivalence de différentes approches grâce à des changements de système de référence. Cette équivalence est manifeste dans le cadre d'un formalisme covariant qui est brièvement exposé. Le choix du système de référence dans lequel les atomes ne sont pas accélérés, mais où le contenu spectral de la lumière et le terme de pompage atomique sont modifiés en conséquence, conduit aux calculs les plus simples. Ce choix permet l'utilisation des diagrammes de matrice densité habituels ainsi que des règles diagrammatiques associées. Des exemples sont explicitement calculés dans le cas d'une onde de gravitation sinusoïdale. Finalement, on présente une brève discussion du rapport signal sur bruit des raies latérales induites par les ondes de gravitation sur les résonances de saturation.
\end{abstract}

\footnotetext{
Abstract. - We present a general framework in which non-linear optical phenomena for an ensemble of accelerated atoms can be studied. We show the equivalence of various approaches through frame transformations. This equivalence is understood within the context of a covariant formalism which is briefly sketched. The choice of the frame, in which the atoms are not accelerated but where the light spectrum and the atomic source term are correspondingly modified, leads to the simplest calculation. It enables one to use the usual density matrix diagrams and associated rules. Explicit examples are given in the case of a sinusoidal gravitational wave. Finally a brief discussion of the signal-to-noise ratio of the sidebands induced by gravitational waves on saturation resonances is outlined.
} 
It has been suggested that the non-linear response of an ensemble of independent two-level atoms (or molecules) submitted to a strong monochromatic electromagnetic wave at $\omega_{1}$, and probed by a second collinear wave at $\omega_{2}$ could be a sensitive detector of gravitational waves [1].

The Einstein equivalence principle suggests various quasi-Newtonian approaches to this problem which use different frames of reference. For instance one can choose a frame in which the laser is at rest and the atoms are accelerated or conversely a frame in which the atoms are not accelerated but where the laser is accelerated which yields a Doppler shift of its frequency. However these various approaches did not seem to agree on the expected orders of magnitude of the effect [2].

Our goal has been to check these various calculations, to reconcile the points of view and to provide a general framework to treat non-linear optical phenomena in the presence of a gravitational field.

If one interprets the gravitational field within the context of Newtonian physics (as generating an acceleration $\mathbf{a}(\mathbf{x}, \mathbf{v}, t)$ ), the following equation can be shown to hold, both for the Wigner function and for the density matrix operator when the motion is treated classically [3] (quantum recoil effects can be incorporated following [4]) :

$$
\left[\frac{\partial}{\partial t}+\mathbf{v} \cdot \boldsymbol{\nabla}+\mathbf{a} \cdot \nabla_{\mathbf{v}}\right] \rho_{\alpha \beta}(\mathbf{x}, \mathbf{v}, t)=\lambda_{\alpha \beta}(\mathbf{x}, \mathbf{v}, t)-\left(i \omega_{\alpha \beta}+\gamma_{\alpha \beta}\right) \rho_{\alpha \beta}+\frac{1}{i \hbar} \sum_{\gamma}\left[V_{\alpha \gamma} \rho_{\gamma \beta}-\rho_{\alpha \gamma} V_{\gamma \beta}\right]
$$

where $\alpha, \beta, \gamma \ldots$ denote internal energy states.

One can use a Liouville space notation for the internal degrees of freedom, then $\rho_{\alpha \beta}$ appears as a vector $\mid \rho(\mathbf{x}, \mathbf{v}, t) \gg$ :

$$
\left[\frac{\partial}{\partial t}+\mathbf{v} \cdot \nabla+\mathbf{a} \cdot \nabla_{\mathbf{v}}\right]|\rho \gg=| \Lambda \gg-i\left(L_{0}+i R\right)\left|\rho \gg-i L_{\mathrm{int}}\right| \rho \gg .
$$

In these equations $\mathbf{v}$ is the velocity; the pumping source is represented by $\lambda_{\alpha \beta}$ in equation 1 and by $\mid \Lambda \gg$ in equation $2 ; L_{0}$ is the internal Liouvillian with eigenvalues $\omega_{\alpha \beta} ; R$ is a relaxation super-operator with eigenvalues $-\gamma_{\alpha \beta}$. The coupling between the dipole moment operator $\mu$ and the electric field of the lasers $\boldsymbol{E}$ is taken into account through the last terms of equations 1 and 2 where $V_{\alpha \beta}(\mathbf{x}, t)=-\mu_{\alpha \beta} . \&(\mathbf{x}, t)$.

The formal solution of equations 1 or 2 can be written as :

$$
\left|\rho(\mathbf{x}, \mathbf{v}, t) \gg=\int_{-\infty}^{t} \mathrm{~d} t_{0} \mathcal{C} \exp \left\{-\int_{t_{0}}^{t}\left(i L_{0}-R+i L_{\text {int }}+\mathbf{v} \cdot \nabla+\mathbf{a} \cdot \nabla_{\mathbf{v}}\right) \mathrm{d} t^{\prime}\right\}\right| \Lambda\left(\mathbf{x}, \mathbf{v}, t_{0}\right) \gg
$$

where $\mathfrak{G}$ denotes the chronological product.

Using the definition of $\mathcal{C}[5]$ we rewrite (3) as an integral equation :

$$
|\rho(t) \gg=| \rho^{(0)}(t) \gg-i \int_{-\infty}^{t} \mathrm{~d} t^{\prime} \mathcal{U}\left(t, t^{\prime}\right) L_{\mathrm{int}}\left(t^{\prime}\right) \mid \rho\left(t^{\prime}\right) \gg
$$

where $U\left(t, t^{\prime}\right)$ is the evolution operator :

$$
\mathcal{U}\left(t, t^{\prime}\right)=\mathscr{C} \exp \left\{-\int_{t^{\prime}}^{t}\left(i L_{0}-R+\mathbf{v} \cdot \nabla+\mathbf{a} \cdot \nabla_{v}\right) \mathrm{d} t^{\prime \prime}\right\}
$$

and where $\mid \rho^{(0)} \gg$ is the density operator in the absence of laser fields. 
The interest of equation 4 in quantum optics calculations is that it can easily be iterated from $\left.\left|\rho^{(0)}\right\rangle\right\rangle$ to obtain the non-linear response to any desired order in the strength of the electromagnetic field. The only difficulty here comes from the non-commuting character of the $\mathbf{v} \cdot \nabla$ and $\mathbf{a} \cdot \nabla_{\mathbf{v}}$ operators in (5). Two methods can be used :

a) The solution can be expanded in a perturbation series with respect to a. For this purpose we proceed as before with the chronological product :

$$
\begin{array}{r}
|\rho(t)\rangle=\left|\rho^{(00)}(t)\right\rangle-\int_{-\infty}^{t} \mathrm{~d} t_{1} \exp \left\{-\left(i L_{0}-R+\mathbf{v} \cdot \nabla\right)\left(t-t_{1}\right)\right\} \times \\
\left.\times\left[\mathbf{a}\left(t_{1}\right) \cdot \nabla_{\mathbf{v}}+i L_{\text {int }}\left(t_{1}\right)\right]\left|\rho\left(t_{1}\right)\right\rangle\right\rangle
\end{array}
$$

where $\left|\rho^{(00)}\right\rangle$ is the density operator in the absence of both electromagnetic and gravitational fields from which equation 6 can be iterated to any order with respect to both fields. This is the basis of the calculation of references $[1,6]$. It has the disadvantage to require an additional vertex of interaction (with the gravitational field) in the density matrix diagrams [6] and the calculations are rather cumbersome.

b) When a can be considered as independent of space in the atomic propagator (excellent approximation which is also used in the previous calculation) the operator $U$ can be rigourously expressed by a second-order Magnus expansion [7] as the product of space and velocity translation operators :

$$
\begin{aligned}
u\left(t, t^{\prime}\right)=\exp \left\{-\left(i L_{0}-R\right.\right. & \left.+\mathbf{v} \cdot \nabla)\left(t-t^{\prime}\right)\right\} \times \\
& \times \exp \left\{\int_{t^{\prime}}^{t} \mathrm{~d} t_{1}\left(t_{1}-t^{\prime}\right) \mathbf{a}\left(t_{1}\right) \cdot \nabla\right\} \exp \left\{-\int_{t^{\prime}}^{t} \mathrm{~d} t_{1} \mathbf{a}\left(t_{1}\right) \cdot \nabla_{\mathbf{v}}\right\}
\end{aligned}
$$

from which we obtain the integral equation :

$$
\begin{aligned}
& |\rho(\mathbf{x}, \mathbf{v}, t)\rangle=-i \int_{0}^{+\infty} \mathrm{d} \tau \mathrm{e}^{-\left(i L_{0}-R\right) \tau} \times \\
& \times\left[L_{\text {int }}(\mathbf{x}-\Delta \mathbf{x}, t-\tau)|\rho(\mathbf{x}-\Delta \mathbf{x}, \mathbf{v}-\Delta \mathbf{v}, t-\tau)\rangle+i|\Lambda(\mathbf{x}-\Delta \mathbf{x}, \mathbf{v}-\Delta \mathbf{v}, t-\tau)\rangle\right]
\end{aligned}
$$
with

$$
\Delta \mathbf{x}=\mathbf{v} \tau-\int_{t-\tau}^{t} \mathrm{~d} t_{1} \mathbf{a}\left(t_{1}\right)\left(t_{1}-t+\tau\right), \quad \Delta \mathbf{v}=\int_{t-\tau}^{t} \mathrm{~d} t_{1} \mathbf{a}\left(t_{1}\right)
$$

Density matrix diagrams comprising only electromagnetic vertices can be drawn as in [4] with the previous rules for assigning space-time coordinates to successive vertices. (Thêse diagrams have been used successfully to compute the linear and 3-order response.)

Equation 8 can also be obtained as the integral form of an equation, identical to (2) but with $\mathbf{a}=0$, followed by a change of independent variables :

$$
\begin{aligned}
\left(\frac{\partial}{\partial t^{\prime}}+\mathbf{v}^{\prime} \cdot \nabla^{\prime}\right)\left|\rho^{\prime}\left(\mathbf{x}^{\prime}, \mathbf{v}^{\prime}, t^{\prime}\right)\right\rangle= & \left|\Lambda^{\prime}\left(\mathbf{x}^{\prime}, \mathbf{v}^{\prime}, t^{\prime}\right)\right\rangle- \\
& \left.-i\left(L_{0}+i R\right)\left|\rho^{\prime}\left(\mathbf{x}^{\prime}, \mathbf{v}^{\prime}, t^{\prime}\right)\right\rangle-i L_{\text {int }}^{\prime}\left(\mathbf{x}^{\prime}, t^{\prime}\right)\left|\rho^{\prime}\left(\mathbf{x}^{\prime}, \mathbf{v}^{\prime}, t^{\prime}\right)\right\rangle\right\rangle
\end{aligned}
$$


with

$$
\begin{aligned}
& \mathbf{x}=\mathbf{X}\left(\mathbf{x}^{\prime}, \mathbf{v}^{\prime}, t\right)=\mathbf{x}^{\prime}+\int_{0}^{t^{\prime}} \mathrm{d} t_{1} \int_{0}^{t_{1}} \mathrm{~d} t_{2} \mathbf{a}\left(t_{2}\right)=\mathbf{x}^{\prime}+\int_{0}^{t^{\prime}} \mathrm{d} t_{1}\left(t^{\prime}-t_{1}\right) \mathbf{a}\left(t_{1}\right), \\
& t=T\left(\mathbf{x}^{\prime}, \mathbf{v}^{\prime}, t^{\prime}\right)=t^{\prime} \\
& \mathbf{v}=\mathbf{V}\left(\mathbf{x}^{\prime}, \mathbf{v}^{\prime}, t^{\prime}\right)=\mathbf{v}^{\prime}+\int_{0}^{t^{\prime}} \mathbf{a}\left(t_{1}\right) \mathrm{d} t_{1}
\end{aligned}
$$

and

$$
\begin{gathered}
\left.\left|\rho^{\prime}\left(\mathbf{x}^{\prime}, \mathbf{v}^{\prime}, t^{\prime}\right)\right\rangle=|\rho(\mathbf{x}, \mathbf{v}, t)\rangle\right\rangle \\
L_{\text {int }}^{\prime}\left(\mathbf{x}^{\prime}, t^{\prime}\right)=L_{\text {int }}(\mathbf{x}, t)=L_{\text {int }}\left(\mathbf{X}\left(\mathbf{x}^{\prime}, \mathbf{v}^{\prime}, t^{\prime}\right), T\left(\mathbf{x}^{\prime}, \mathbf{v}^{\prime}, t^{\prime}\right)\right) \\
\left|\Lambda^{\prime}\left(\mathbf{x}^{\prime}, \mathbf{v}^{\prime}, t^{\prime}\right)\right\rangle=|\Lambda(\mathbf{x}, \mathbf{v}, t)\rangle=\mid \Lambda\left(\mathbf{X}\left(\mathbf{x}^{\prime}, \mathbf{v}^{\prime}, t^{\prime}\right), \mathbf{V}\left(\mathbf{x}^{\prime}, \mathbf{v}^{\prime}, t^{\prime}\right), T\left(\mathbf{x}^{\prime}, \mathbf{v}^{\prime}, t^{\prime}\right)\right\rangle .
\end{gathered}
$$

Since it is clear from equation 11 that the phase space element is preserved, equation 10 corresponds physically to a description in an accelerated frame of reference in which the atom undergoes no acceleration and where the effect of gravitation comes in, as a modulation of the laser field in (13) and as a suitable modification of the pumping operator (14).

The previous analysis suggests three comments :

1) The consistency of the two Newtonian-like approaches to the problem is most clearly understood within the context of a covariant formalism in which the following replacement rules are applied :

$$
\begin{array}{clrl}
\frac{\partial}{\partial t}+\mathbf{v} \cdot \nabla+\mathbf{a} \cdot \nabla_{\mathbf{v}} & \rightarrow u^{\mu} \partial_{\mu}-\Gamma_{\rho \sigma}^{i} u^{\rho} u^{\sigma} \frac{\partial}{\partial u^{i}} ; & \rho, \mu & =0,1,2,3 \\
V_{\alpha \beta} \rightarrow \mu_{\alpha \beta}^{\hat{i}} e_{i}^{\rho} F_{\rho \lambda} u^{\lambda} & i=1,2,3
\end{array}
$$

where $u^{\mu}$ is the 4-velocity, $\Gamma_{\rho \sigma}^{i}=\frac{1}{2} g^{i \tau}\left\{g_{\rho \tau, \sigma}+g_{\sigma \tau, \rho}-g_{\rho \sigma, \tau}\right\}$ is the connection, $F_{\rho \lambda}$ the Maxwell tensor and $e_{\sigma}^{\rho}$ a tetrad which is equal to $\delta_{\sigma}^{\rho}$ in the rest frame of the atom before the arrival of the gravitational wave and which undergoes a parallel transport when the gravitational wave is present.

2) Calculations are much simpler to perform in a frame where the atom is in uniform motion or at rest and where the light spectrum is correspondingly modified. Then usual diagrammatic rules can be applied [4].

3) One should be very careful in specifying the boundary condition implicit in the pumping operator since a Maxwell-Boltzmann distribution or an ideal molecular beam are not invariant concepts in a frame transformation such as (11). This last point is at the origin of past discrepancies between various calculations.

As the first example we consider the linear absorption term described by diagram 1 with an acceleration a corresponding to a monochromatic gravitational wave of amplitude $h$ and circular frequency $\xi$. The component of a along the optical $z$-axis will be written as :

$$
(h / 2) z \xi^{2} \cos \xi t
$$

where $z$ is the distance between the laser and the atom to be considered $\left({ }^{1}\right)$. If the atomic source

( $\left.{ }^{1}\right)$ This acceleration is specified in a Fermi reference system where the laser is in free fall (i.e. properly suspended!). 
term is :

$$
\lambda_{a}^{\prime}\left(\mathbf{v}^{\prime}\right)=r_{a} \delta\left(\mathbf{v}^{\prime}-\mathbf{v}_{0}\right)
$$

(ideal beam in a frame where the atom is not accelerated) the change in power absorbed per unit volume from the probe beam averages in time to zero except when $\omega_{2}=\omega_{1} \pm \xi$. When $\omega_{2}=\omega_{1}-\xi$ we find :

$$
\begin{aligned}
& \frac{\mathrm{d} W_{2}}{\mathrm{~d} V}=\hbar \omega_{2}\left(\frac{r_{a}}{\gamma_{a}}\right) \Omega_{2} \Omega_{1} \frac{h k z}{2} \operatorname{Re}\left\{i \mathrm{e}^{i\left(\varphi_{2}-\varphi_{1}\right)} \times\right. \\
& \left.\quad \times\left(\left[\gamma_{b a}+i\left(\omega_{b a}+\delta-\gamma \omega_{1}+\mathbf{k} \cdot \mathbf{v}_{0}\right)\right]^{-1}-\left[\gamma_{b a}+i\left(\omega_{b a}+\delta+\xi-\gamma \omega_{1}+\mathbf{k} \cdot \mathbf{v}_{0}\right)\right]^{-1}\right)\right\}
\end{aligned}
$$

where the 1rst term comes from the polarization created by the carrier at $\omega_{1}$ heterodyned by the probe side band at $\omega_{2}+\xi$ and where the 2nd term comes from the polarization created by the pump side band at $\omega_{1}-\xi$ heterodyned by the probe carrier at $\omega_{2}$. In this expression $\Omega_{2}$ and $\Omega_{1}$ are Rabi frequencies, $\varphi_{2}$ and $\varphi_{1}$ field phases; the recoil shift $\delta=\hbar k^{2} / 2 M$ and $\gamma=\left(1-v^{2} / c^{2}\right)^{-1 / 2}$ have been introduced as in reference 4.

For $\omega_{1}$ tuned to resonance we obtain :

$$
\frac{\mathrm{d} W_{2}}{\mathrm{~d} V}=-\left(\frac{h k z}{2}\right)\left[\frac{\hbar \omega_{2}}{T_{1}}\left(\frac{r_{a}}{\gamma_{a}}\right)\right]\left(\frac{\Omega_{2}}{\Omega_{1}}\right)\left(\frac{\Omega_{1}^{2} T_{1}}{\gamma_{b a}}\right) \operatorname{Re}\left[\frac{\xi}{\gamma_{b a}+i \xi} \mathrm{e}^{i\left(\varphi_{2}-\varphi_{1}\right)}\right] .
$$

where we have introduced $T_{1}=\frac{1}{2}\left(\frac{1}{\gamma_{a}}+\frac{1}{\gamma_{b}}\right)$ relaxation time for energy in order to display factors having an obvious physical interpretation :

$\frac{\hbar \omega_{2}}{T_{1}}\left(\frac{r_{a}}{\gamma_{a}}\right)$ is the maximum rate at which energy can be exchanged with the thermal bath. $\left(\frac{\Omega_{1}^{2} T_{1}}{\gamma_{b a}}\right)$ is an excitation efficiency (one fourth of the saturation parameter $S$ ) which may reach values of $\sim 1$ beyond which the absorption gets strongly saturated.

$\Omega_{2} / \Omega_{1}$ is a heterodyne factor which for the same reason cannot be larger than one.

The last factor is proportional to $\xi$ for small values of $\xi\left(\frac{\xi}{\gamma_{b a}} \cos \left(\varphi_{2}-\varphi_{1}\right)\right)$ and it can be of the order of 1 for $\xi \gg \gamma_{b a}$.

$\mathrm{d} W_{2} / \mathrm{d} V$ is thus at best of the order of the usual absorbed power times the modulation index $h k z / 2$.

Expression 18 averaged over a Maxwell-Boltzmann distribution of the velocities $\mathbf{v}_{0}$ (cell case) gives :

$$
\frac{\mathrm{d} W_{2}}{\mathrm{~d} V}=-2\left(\frac{h k z}{2}\right)\left[\frac{\hbar \omega_{2}}{T_{1}}\left(\frac{r_{a}}{\gamma_{a}}\right)\right]\left(\frac{\Omega_{2}}{\Omega_{1}}\right)\left(\frac{\Omega_{1}^{2} T_{1}}{\gamma_{b a}}\right)\left(\frac{\gamma_{b a}}{k u}\right)\left(\frac{\xi}{k u}\right) \cos \left(\varphi_{2}-\varphi_{1}\right)
$$

where $k u$ is the Doppler width ( $u=\sqrt{2 k_{\mathrm{B}} T / M}$ is the most probable velocity of atoms).

Comparing (20) and (19) we discover that two new factors have appeared :

$\gamma_{b a} / k u$ is the fraction of atoms used in the velocity distribution along the optical axis (relative width of the hole burnt in this velocity distribution [8]).

$\xi / k u$ appears because the modulation frequency is, in this case, much smaller than the Doppler line width $k u$.

Since these two factors are very small, the signal is, in this case, much smaller than in the beam case. 
Since authors of reference 1 considered a Maxwell-Boltzmann distribution in a frame where the atom is accelerated we shall consider this case now and start with the ideal beam condition :

$$
\lambda_{a}=r_{a} \delta\left(\mathbf{v}-\mathbf{v}_{0}\right)
$$

which yields a time dependent velocity distribution in the "primed frame ".

This results in an additional term for $\mathrm{d} W_{2} / \mathrm{d} V$ which is :

$$
\hbar \omega_{2}\left(\frac{r_{a}}{\gamma_{a}}\right) \Omega_{2} \Omega_{1}\left(\frac{h k z}{2}\right) \operatorname{Re}\left[\mathrm{e}^{i\left(\varphi_{2}-\varphi_{1}\right)}\left\{\frac{\gamma_{a}}{\gamma_{a}+i \xi} \cdot \frac{\xi}{\left[\gamma_{b a}+i\left(\omega_{b a}+\delta+\xi-\gamma \omega_{1}+\mathbf{k . v _ { 0 } )}\right]^{2}\right.}\right\}\right] .
$$

After averaging the overall result over the Maxwell-Boltzmann distribution we find in this case :

$$
\frac{\mathrm{d} W_{2}}{\mathrm{~d} V}=-2\left(\frac{h k z}{2}\right)\left[\frac{\hbar \omega_{2}}{T_{1}}\left(\frac{r_{a}}{\gamma_{a}}\right)\right]\left(\frac{\Omega_{2}}{\Omega_{1}}\right)\left(\frac{\Omega_{1}^{2} T_{1}}{\gamma_{b a}}\right)\left(\frac{\gamma_{b a}}{k u}\right)\left(\frac{\xi}{k u}\right) \operatorname{Re}\left[\frac{i \xi \mathrm{e}^{i\left(\varphi_{2}-\varphi_{1}\right)}}{\gamma_{a}+i \xi}\right]
$$

which is identical to the result of reference 1 apart from a constant factor but which differs from (20) in general. If $\xi \gg \gamma_{b a}$ the two results are identical since then the equilibrium population cannot follow the changes in the velocity distribution in the " primed frame » and the additional term does not contribute.

As the second example we consider the non-linear term corresponding to diagram 2 with :

$$
\lambda_{a}^{\prime}=r_{a} F\left(\mathbf{v}^{\prime}\right) \text { and } \omega_{2}=\omega_{1}+\xi .
$$

This diagram is calculated as in [4] and for a Maxwellian velocity distribution we find :

$$
\begin{aligned}
\frac{\mathrm{d} W_{2}}{\mathrm{~d} V} & =2 \sqrt{\pi}\left(\frac{h k z}{2}\right)\left[\frac{\hbar \omega_{2}}{T_{1}}\left(\frac{r_{a}}{\gamma_{a}}\right)\right]\left(\frac{\Omega_{2}}{\Omega_{1}}\right)\left(\frac{\Omega_{1}^{2} T_{1}}{\gamma_{b a}}\right)^{2}\left(\frac{\gamma_{b a}}{k u}\right) \operatorname{Re}\left\{i \mathrm{e}^{-i\left(\varphi_{2}-\varphi_{1}\right)} \times\right. \\
\times & \left.\frac{\gamma_{b a}}{T_{1}}\left[f\left(2 \gamma_{b a}, \gamma_{b}\right)-f\left(2 \gamma_{b a}+i \xi, \gamma_{b}\right)-f\left(2 \gamma_{b a}+i \xi, \gamma_{b}+i \xi\right)+f\left(2 \gamma_{b a}+2 i \xi, \gamma_{b}+i \xi\right)\right]\right\}
\end{aligned}
$$

where $f(x, y)=1 / x y$ in the case of plane waves but may be a more complicated line shape such as those calculated in references 3 and 4 to include the effect of transit-broadening in a Gaussian beam (see equation (105) of Ref. 3) or Ramsey fringes.

The above result is consistent with that of references 1,6 and 10 . To prove the identity with that of reference 1 (except for a constant factor) we used a term by term identification which was made possible thanks to the above (b) procedure. The following identity found in this process can be used to show the equivalence of the two results :

$$
\begin{aligned}
-\frac{1}{2 \gamma_{b a} \gamma_{b}}+\frac{1}{2 \gamma_{b a}+i \xi} \cdot \frac{1}{\gamma_{b}}+\frac{1}{2 \gamma_{b a}+i \xi} \cdot \frac{1}{\gamma_{b}+i \xi}-\frac{1}{2 \gamma_{b a}+2 i \xi} \cdot \frac{1}{\gamma_{b}+i \xi}= \\
=\frac{\xi^{2}}{\left(2 \gamma_{b a}+i \xi\right)^{2}}\left[\frac{1}{2 \gamma_{b a} \gamma_{b}}+\frac{1}{\gamma_{b}\left(\gamma_{b}+i \xi\right)}+\frac{1}{\left(\gamma_{b}+i \xi\right)\left(2 \gamma_{b a}+2 i \xi\right)}\right] .
\end{aligned}
$$

The expression 24 was obtained by a much simpler calculation with all the advantages of the diagrammatic techniques already developed for high resolution non-linear spectroscopy. Furthermore each term has a simple physical interpretation and corresponds to a different choice of each one of the four vertices for the interaction with the gravitation-induced sideband of the laser light. 
The result (24) is independent of the frame in which $F(v)$ is assumed. This is because the light beam performs its own velocity selection through the inhomogeneous saturation process (hole burning) independently of the equilibrium velocity distribution as long as the width of this distribution is large compared to $\gamma_{b a} / k$ (infinite Doppler width approximation). The comparison with the ideal beam case (formula 19) shows that for a saturation parameter close to unity the only loss in signal comes from the factor $\gamma_{b a} / k u$ which expresses the fact that the formation rate $r_{a}$ is relative to the overall Maxwell-Boltzmann distribution and that only the small fraction of atoms corresponding to the hole burnt in velocity space can be used. The comparison with linear spectroscopy in the cell case shows that the factor $\xi / k u$ has disappeared. The saturation method is therefore completely equivalent to an experiment performed with a beam.

Let us finally give a brief discussion of the sensitivity of the method. The signal-to-noise ratio corresponding to shot-noise-limited optical detection is limited by saturation $(S=1)$ or by the $\pi$-pulse condition [9] to the maximum value : $\pi C(2 \eta)^{1 / 2}\left(w_{0} / \lambda\right)\left(\tau \tau_{R} / T_{1} T_{2}\right)^{1 / 2}$ in the collision regime or $\pi^{3 / 2} C(2 \eta)^{1 / 2}(u / \lambda)\left(\tau \tau_{R}\right)^{1 / 2}$ in the free-flight regime where $C$ is the signal contrast (ideally 1 in a pure optical experiment and $h k L$ in our case $\left({ }^{2}\right)$ ); $\eta$ is the detector efficiency, $w_{0}$ the beam waist radius, $\tau$ the integration time, $\tau_{\mathrm{R}}$ the reciprocal of the Einstein $A$ coefficient for the considered two-level system, $T_{1}$ and $T_{2}$ the relaxation times for energy and for the optical coherence $\left(T_{2}=1 / \gamma_{b a}\right)$.

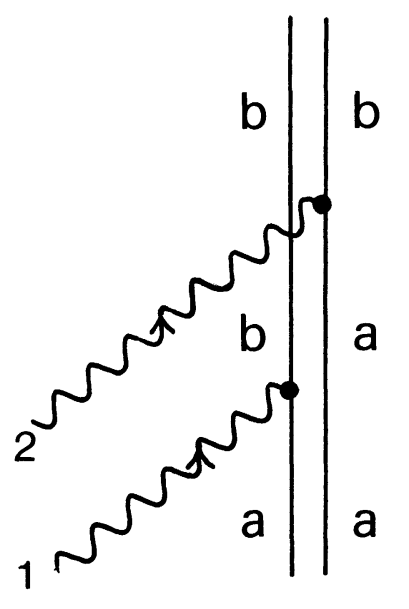

(1)

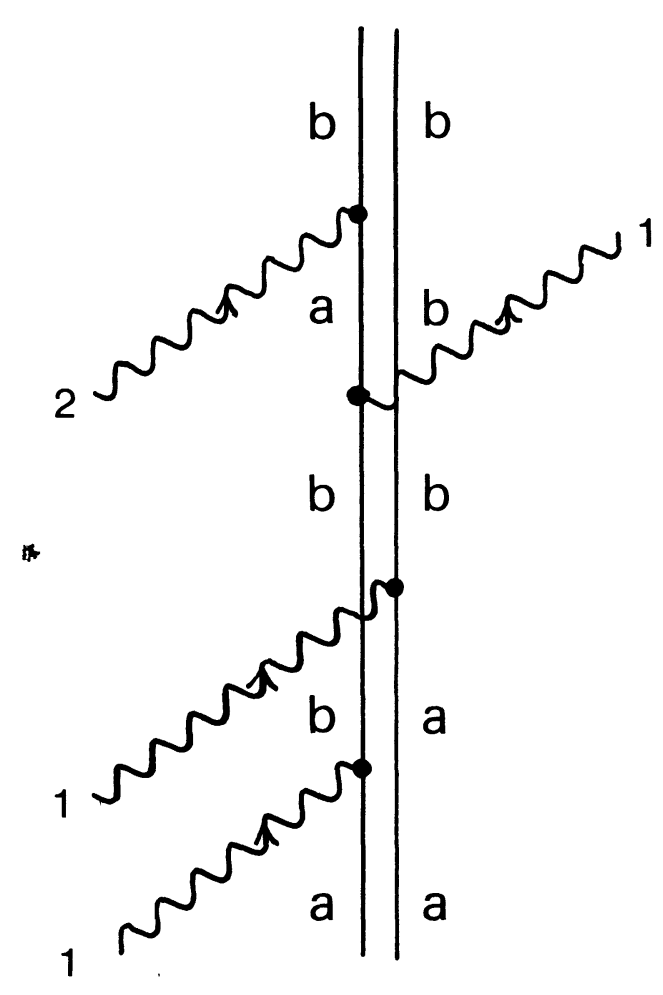

(2)

( $\left.{ }^{2}\right) L$ is the distance between the laser and the centre of the cell (i.e. the spatial average value of $z$ ). 
This signal-to-noise ratio can be at best of the order of $10^{9} h k L$ for $\tau=1 \mathrm{~s}$ (with $u / \lambda \sim 10^{9} \mathrm{~Hz}$ in the visible and $\left.\pi^{3} \tau_{\mathrm{R}} \sim 1 \mathrm{~s}\right)$. For $k L \sim 10^{12}(L \sim 100 \mathrm{~km})$, this would correspond to $h_{\text {min. }} \sim 10^{-21}$.

As a conclusion we 'emphasize the fact that the results obtained yield a covariant consistent picture of non-linear optics in a gravitational field and provide a tool for studying accelerated atomic systems within the frame-work of Newtonian physics.

\section{References}

[1] Nesterikhin, Y. E., Rautian, S. G. and Smirnov, G. I., J.E.T.P. 48 (1978) 1.

[2] Scully, M. O., Private communication, Bad-Windsheim, Aug. 1981.

[3] Bordé, Ch. J., Hall, J. L., Kunasz, C. V. and Hummer, D. G., Phys. Rev. A 14 (1976) 236.

[4] BORDÉ, Ch. J., Density matrix equations and diagrams for high resolution non-linear spectroscopy in advances in laser spectroscopy NATO ASI Series (Plenum Press) 1983.

[5] LANDAU, L. and LIFCHITZ, E., Théorie quantique relativiste, MIR, 1972.

[6] Brillet, A. and Tourrenc, Ph., in Rayonnement gravitationnel N. Deruelle and T. Piran Editors (North Holland) 1983.

[7] Pechukas, P. and Light, J. C., J. Chem. Phys. 44 (1966) 3897.

[8] See for example Sargent, M., Scully, M. O. and LAMB, W. E., in Laser Physics (Addison and Wesley) 1974.

[9] BoRdÉ, Ch. J., in Proceedings of the Conference on Photoacoustic and Photothermal spectroscopy, J. Physique Colloq. 44 (1983) C6-593.

[10] Tourrenc, Ph., Damour, Th., Sharma, J. and Bordé, Ch. J., in Contributed papers of the 10th international conference on General relativity and gravitation, Padova July 1983, B. Bertotti, F. de Felice, A. Pascolini editors. 